Pacific Journal of Mathematics

CLOSED RANGE THEOREMS FOR CONVEX SETS AND 


\title{
CLOSED RANGE THEOREMS FOR CONVEX SETS AND LINEAR LIFTINGS
}

\author{
T. ANDO
}

Let $M$ be a closed subspace of a Banach space $E$ such that its annihilator $M^{\perp}$ is the range of a projection $P$. Given a closed convex subset $S$ containing 0 , the first problem of this paper is to find a condition for $\tau(S)$ to be closed where $\tau$ is the canonical map from $E$ to $E / M$. Closure is guaranteed if $S$ is splittable in the sense that the polar $S^{0}$ coincides with the norm-closed convex hull of $P\left(S^{0}\right) \cup Q\left(S^{0}\right)$, where $Q=1-$ $P$. The second problem is to give a condition for existence of a linear map $\varphi$, called a linear lifting, from $E / M$ to $E$ such that $\tau \circ \varphi=1$ and $\varphi \circ \tau(S) \leqq S$. A linear lifting exists if and only if $M$ is the kernel of a projection making $S$ invariant. Of special interest is the case where $S$ is a ball or a cone. When the unit ball is splittable, existence of a linear lifting of norm one is guaranteed under suitable conditions on $E / M$, which are satisfied by separable $L_{p}$ and $C(X)$ on compact metrizable $X$. If further $E$ is an ordered Banach space, and if both $P$ and $Q$ are positive, $M$ is shown to be the kernel of a positive projection of norm one.

Though the closed range theorem (Theorem 1) yields immediately an abstract version of the Rudin-Carleson-Bishop theorem on normpreserving extensions of functions defined on a peak set, in $\S 2$ further modification (Theorem 2) is shown to include Gamelin's extension [5] of the Rudin-Carleson-Bishop theorem in abstract form. Recently a different approach to generalization of the Gamelin theorem was made by Alfsen and Hirsberg [1]. In $\S 3$ it is indicated how the closed range theorem is applied to give unified proofs for results of Davies [4] and Perdrizet [9] on closedness of a cone in a quotient space and on order-preserving extensions. In $\S 4$ an idea of Pełczynski-Michael [8] is further developed for the closed range theorem to produce existence of linear liftings under suitable conditions. The PełczynskiMichael theorems are generalized in abstract form (Theorems 5 and 6).

1. Preliminary. Let $E$ be a real or complex Banach space with unit ball $U . E^{*}$ and $E^{* *}$ are its dual and second dual respectively, and $E$ is always imbedded canonically into $E^{* *} . \quad x, y, z, \cdots$ are vectors in $E$ or $E^{* *}$ while $f, g, h, \cdots$ are functionals in $E^{*}$. For $x \in E^{* *}$ and $f \in E^{*} f(x)$ is used instead of $x(f)$. The weak topology $\sigma\left(E^{*}, E\right)$ on $E^{*}$ is called the weak $k^{*}$ topology while $\sigma\left(E^{* *}, E^{*}\right)$ on $E^{* *}$ is the $w_{e a k^{* *}}$ topology. For a subset $S$ of $E$ its norm-closure and its weak** 
closure (in $E^{* *}$ ) are denoted by $\bar{S}$ and $S^{\sim}$ respectively.

The polar $S^{0}$ is defined as the set of all $f$ such that $\operatorname{Re} f(x) \leqq 1$ or $f(x) \leqq 1$ on $S$ according as the scalar field is complex or real. When $S$ is a subspace its polar coincides with its annihilator $S^{\perp}$ consisting of all $f$ vanishing on it. The following basic facts are used frequently in this paper. Proofs are found, for instance, in [10]. Let $S_{1}$ and $S_{2}$ be closed convex subsets of $E$ containing $0 . \quad\left(S_{1} \cap S_{2}\right)^{0}$ coincides with the weak* closure of conv $\left(S_{1}^{0} \cup S_{2}^{0}\right)$ where conv $(\cdot)$ denotes the convex hull. The weak** closure $S_{1}^{\sim}$ coincides with the polar of $S_{1}^{0}$ in duality $\left\langle E^{* *}, E^{*}\right\rangle$ and $S_{1}=E \cap S_{1}^{\sim}$. Thus coincidence $\left(S_{1} \cap S_{2}\right)^{\sim}=$ $S_{1}^{\sim} \cap S_{2}^{\sim}$ occurs if and only if the weak* and the norm closure of conv $\left(S_{1}^{0} \cup S_{2}^{0}\right)$ coincide. In some case conv $\left(S_{1}^{0} \cup S_{2}^{0}\right)$ becomes itself weak* closed. Here the Krein-Smulian theorem is quite useful: conv $\left(S_{1}^{0} \cup S_{2}^{0}\right)$ is weak* closed if (and only if) $\gamma U^{0} \cap \operatorname{conv}\left(S_{1}^{0} \cup S_{2}^{0}\right)$ is weak* closed for every $0 \leqq \gamma<\infty$. If $S_{1}$ contains 0 in its interior then $S_{1}^{0}$ is weak* compact and the norm closure of conv $\left(S_{1}^{0} \cup S_{2}^{0}\right)$ is weak* closed. If $S_{1}$ is a subspace or a cone, the weak* closure of conv $\left(S_{1}^{0} \cup S_{2}^{0}\right)$ is just that of $S_{1}^{\llcorner}+S_{2}^{0}$. In case both $S_{1}$ and $S_{2}$ are subspaces, $S_{1}^{\perp}+S_{2}^{\perp}$ is weak* closed if and only if $S_{1}+S_{2}$ is norm-closed.

Suppose now that $E$ is a real Banach space provided with a closed proper cone $E_{+}$. $E_{+}$gives rise to natural ordering in $E$ under which it becomes the set of all positive vectors: $x \leqq y$ means $y-x \in E_{+}$. In this respect $E_{+}$is called the positive cone. The dual positive cone $E_{+}^{*}$ is defined as the set of $f$ nonnegative on $E_{+}$, or equivalently $E_{+}^{*}=$ $-E_{+}^{0} \cdot E$ is called an ordered Banach space if $E=E_{+}-E_{+}$and if there is $\gamma<\infty$ with $\left(U-E_{+}\right) \cap\left(U+E_{+}\right) \subseteq \gamma U$. The latter condition is equivalent to that every subset of the form $\left\{x ; y_{1} \leqq x \leqq y_{2}\right\}$ is normbounded. For notational convenience the relation $x \leqq y+\varepsilon$ in an ordered Banach space means that there is $z \geqq 0$ such that $\|z\|<$ $\varepsilon$ and $x \leqq y+z$. An ordered Banach space or its norm is called regular if $\|x\|=\inf \{\|y\| ;-y \leqq x \leqq y\}$ for every $x$. A regular norm is monotone on the positive cone in the sense that $0 \leqq x \leqq y$ implies $\|x\| \leqq\|y\|$. An ordered Banach space admits an equivalent regular norm. In fact, the functional $\|x\|_{0}=\inf \{\|y\| ;-y \leqq x \leqq y\}$ gives a regular norm.

It is known (cf. [2] and [4]) that $E$ is regular if and only if $E^{*}$ is regular. An ordered Banach space is said to have the Riesz interpolation property if for $y_{i} \geqq x_{j}(i, j=1,2, \cdots, n)$ there is $z$ such that $x_{i} \leqq z \leqq y_{i}(i=1,2, \cdots, n)$. A regular ordered Banach space is called a Banach lattice if it is lattice under the ordering. A Banach lattice has the Riesz interpolation property. It is known (cf. [2] and [4]) that $E$ has the Riesz interpolation property if and only if $E^{*}$ is a lattice. A continuous linear operator between ordered Banach spaces is called positive if it transforms a positive cone into another. 
2. Closed range theorems. $E$ is a real or complex Banach space with unit ball $U$ and $M$ is a closed subspace. The canonical map from $E$ to the quotient space $E / M$ is denoted by $\tau$.

Throughout this section it is assumed:

There is a continuous projection $P$ from $E^{*}$ to $M^{\perp}$, and $Q$ stands for $1-P$.

Remark that the adjoint $Q^{*}$ projects $E^{* *}$ onto $M^{\sim}$ but $M$ is not necessarily range of any projection. $S, S_{1}$ and $S_{2}$ will denote closed convex subsets of $E$ containing $0 . S$ is said to be splittable, or more precisely, $P$-splittable if its polar $S^{0}$ coincides with the norm-closure of $\operatorname{conv}\left(P\left(S^{0}\right) \cup Q\left(S^{0}\right)\right)$.

LEMMA 1. The following conditions are equivalent.

(a) $S$ is splittable.

(b) $S^{\sim}=\left\{x \in E^{* *} ; P^{*} x \in S^{\sim}\right.$ and $\left.Q^{*} x \in S^{\sim}\right\}$.

(c) $\theta(f)=\theta(P f)+\theta(Q f)\left(f \in E^{*}\right)$

where $\theta(f)$ is defined by $\theta(f)=\sup \{\operatorname{Re} f(x) ; x \in S\}$.

Proof. Since the polar of $P\left(S^{0}\right)$ (resp. of $Q\left(S^{0}\right)$ ) in $E^{* *}$ coincides with the set $\left\{x \in E^{* *} ; P^{*} x\left(\operatorname{resp} . Q^{*} x\right) \in S^{\sim}\right\}$ equivalence of (a) and (b) is clear (cf. $\S 1$ ).

$(\mathrm{b}) \Longrightarrow(\mathrm{c})$. Obviously $\theta(f)$ can be defined by

$$
\theta(f)=\sup \left\{\operatorname{Re} f(x) ; x \in S^{\sim}\right\} .
$$

Take $x$ and $y$ in $S$. Then by (b) $P^{*} x+Q^{*} y$ belongs to $S^{\sim}$ so that

$$
\operatorname{Re} P f(x)+\operatorname{Re} Q f(y)=\operatorname{Re} f\left(P^{*} x+Q^{*} y\right) \leqq \theta(f),
$$

leading to $\theta(P f)+\theta(Q f) \leqq \theta(f)$. The reverse inequality is obvious.

(c) $\Rightarrow(b)$. Since the functional $\theta$ is nonnegative because of $S \ni 0$, (c) implies $P\left(S^{0}\right) \cup Q\left(S^{0}\right) \subseteq S^{0}$. Therefore $S^{\sim}$ is contained in the set $\{x \in$ $E^{* *} ; P^{*} x \in S^{\sim}$ and $\left.Q^{*} x \in S^{\sim}\right\}$. Take $x$ with $P^{*} x, Q^{*} x \in S^{\sim}$. Then by (c)

$\operatorname{Re} f(x)=\operatorname{Re} P f\left(P^{*} x\right)+\operatorname{Re} Q f\left(Q^{*} x\right) \leqq \theta(P f)+\theta(Q f)=\theta(f)$.

Thus $x$ belongs to the polar of $S^{0}$ in $E^{* *}$.

CoRollary 1. The unit ball $U$ is splittable if and only if $\|f\|=\|P f\|+\|Q f\|\left(f \in E^{*}\right)$.

Corollary 2. If both $S_{1}$ and $S_{2}$ are splittable. and $\left(S_{1} \cap S_{2}\right)^{\sim}=$ $S_{1}^{\sim} \cap S_{2}^{\sim}$, then $S_{1} \cap S_{2}$ is splittable. 
CoRollary 3. A closed subspace (resp. cone) is splittable if and only if its polar is invariant under $P$ (resp. under $P$ and $Q$ ).

Proof. Let $N$ be a closed cone. $P\left(N^{0}\right) \subseteq N^{0}$ and $Q\left(N^{0}\right) \subseteq N^{0}$ implies $N^{0}=P\left(N^{0}\right)+Q\left(N^{0}\right)=\operatorname{conv}\left(P\left(N^{0}\right) \cup Q\left(N^{0}\right)\right)$. If $N$ is further a subspace, $Q\left(N^{0}\right) \subseteq N^{0}$ follows already from $P\left(N^{0}\right) \subseteq N^{0}$.

LEMma 2. If $S_{1}$ and $S_{2}$ are splittable, for any $\varepsilon>0$ and $\rho>0$ the following inclusion relation holds:

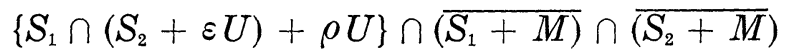

$$
\begin{aligned}
& \subseteq \overline{S_{1} \cap\left(S_{2}+\alpha \varepsilon U\right)+\alpha \rho U \cap M}
\end{aligned}
$$

where $\alpha=\|Q\|$.

Proof. Take any $x$ in the set on the left hand side. Then it follows by splittability that

$$
Q^{*} x \in S_{1}^{\sim} \cap\left(S_{2}^{\sim}+\alpha \varepsilon U^{\sim} \cap M^{\sim}\right)+\alpha \rho U^{\sim} \cap M^{\sim} .
$$

There is $y \in \alpha \rho U^{\sim} \cap M^{\sim}$ such that

$$
Q^{*}(x-y)=Q^{*} x-y \in S_{1}^{\sim} \cap\left(S_{2}^{\sim}+\alpha \varepsilon U^{\sim} \cap M^{\sim}\right)
$$

and

$$
P^{*}(x-y)=P^{*} x \in P^{*}\left(\overline{S_{1}+M}\right) \cap P^{*}\left(\overline{S_{2}+M}\right) \subseteq S_{1}^{\sim} \cap S_{2}^{\sim} .
$$

Then by Lemma $1 x-y \in S_{1}^{\sim}$ and there is $z \in \alpha \varepsilon U^{\sim} \cap M^{\sim}$ such that $x-y-z \in S_{2}^{\sim}$. Finally in view of arguments of $\S 1 x$ belongs to

$$
\begin{aligned}
& E \cap\left\{S_{1}^{\sim} \cap\left(S_{2}^{\sim}+\alpha \varepsilon U^{\sim}\right)+\alpha \rho U^{\sim} \cap M^{\sim}\right\} \\
\subseteq & E \cap\left\{S_{1} \cap\left(S_{2}+\alpha \varepsilon U\right)+\alpha \rho U \cap M\right\}^{\sim} \\
= & \overline{S_{1} \cap\left(S_{2}+\alpha \varepsilon U\right)+\alpha \rho U \cap M} .
\end{aligned}
$$

By definition of the quotient topology $\tau(x)$ belongs to the closure $\overline{\tau(S)}$ if and only if $x$ is contained in $\overline{S+M}$. In particular, $\tau(S)$ is closed if and only if $S+M$ is closed.

LeMma 3. Suppose that $S_{1}$ and $S_{2}$ are splittable. If $\tau(x)$ belongs to $\overline{\tau\left(S_{1}\right)} \cap \overline{\tau\left(S_{2}\right)}$ and $\left\|x-S_{1} \cap S_{2}\right\|<\gamma$ there is $y \in S_{1}$ such that $\tau(x)=$ $\tau(y)$ and $\|x-y\|<\gamma\|Q\|$. In case $\|Q\|=1$ for any $\varepsilon>0$ y can be chosen in $S_{1} \cap\left(S_{2}+\varepsilon U\right)$.

Proof. Let $\alpha=\|Q\|$ and take $\varepsilon^{\prime}$ with $0<\varepsilon^{\prime}<\varepsilon$. By hypothesis $x$ is contained in

$$
\left.\left\{S_{1} \cap\left(S_{2}+\varepsilon^{\prime} U\right)+\gamma^{\prime} U\right\} \cap\left(\overline{S_{1}+M}\right) \cap \overline{\left(S_{2}+M\right.}\right)
$$


for some $\gamma>\gamma^{\prime}>0$. Choose $\varepsilon_{n}>0$ such that $\sum_{n=1}^{\infty} \varepsilon_{n}<\gamma-\gamma^{\prime}$. By Lemma 2 there is $x_{0} \in M$ such that $\left\|x_{0}\right\| \leqq \alpha \gamma^{\prime}$ and $\| x+x_{0}-S_{1} \cap$ $\left(S_{2}+\alpha \varepsilon^{\prime} U\right) \|<\varepsilon_{1}$. Then

$$
x+x_{0} \in \overline{S_{i}+M}+M=\overline{S_{i}+M}
$$

and

$$
x+x_{0} \in S_{1} \cap\left(S_{2}+\alpha \varepsilon^{\prime} U\right)+\varepsilon_{1} U \text {. }
$$

Now inductive procedure based on Lemma 2 makes it possible to find a sequence $\left\{x_{n}\right\}$ in $M$ such that $\left\|x_{n}\right\| \leqq \alpha \varepsilon_{n}$ and $\| x+\sum_{i=0}^{n-1} x_{i}-S_{1} \cap$ $\left(S_{2}+\alpha^{n} \varepsilon^{\prime} U\right) \|<\varepsilon_{n}$. Since $\sum_{n=0}^{\infty}\left\|x_{n}\right\|<\infty, y=x+\sum_{n=0}^{\infty} x_{n}$ is well defined. Obviously $y$ belongs to $S_{1}$, and in case $\alpha=1$, to $S_{1} \cap\left(\overline{S_{2}+}\right.$ $\overline{\left.\varepsilon^{\prime} U\right)} \leqq S_{1} \cap\left(S_{2}+\varepsilon U\right)$. Finally $\tau(x)=\tau(y)$ and $\|x-y\| \leqq \alpha \sum_{n=1}^{\infty} \varepsilon_{n}+$ $\alpha \gamma^{\prime}<\alpha \gamma$.

Now the main result of this paper is near at hand.

THEOREM 1. Suppose that the annihilator of a closed subspace $M$ is the range of a projection $P$ and that $S, S_{1}$ and $S_{2}$ are closed convex subsets containg 0 . Then (a) the image of $S$ under the canonical map $\tau$ from $E$ to $E / M$ is closed whenever $S$ is splittable. (b) If both $S_{1}$ and $S_{2}$ are splittable and $\left(S_{1} \cap S_{2}\right)^{\sim}=S_{1}^{\sim} \cap S_{2}^{\sim}$ then $\tau\left(S_{1}\right) \cap \tau\left(S_{2}\right)=$ $\tau\left(S_{1} \cap S_{2}\right)$. (c) If both $S_{1}$ and $S_{2}$ are splittable and $\|1-P\| \leqq 1$, then the inclusion $\tau\left(S_{1}\right) \cap \tau\left(S_{2}\right) \leqq \tau\left(S_{1} \cap\left(S_{2}+\varepsilon U\right)\right)$ holds for any $\varepsilon>0$.

Proof. (a) follows from Lemma 3 with $S_{1}=S_{2}=S$. Also (c) is a direct consequence of Lemma 3. (b) $S_{1} \cap S_{2}$ is splittable by Corollary 2 and $S_{1} \cap S_{2}+M$ is closed by (a). Now since by hypothesis

$$
\begin{aligned}
& P^{*}\left\{\left(S_{1}+M\right) \cap\left(S_{2}+M\right)\right\} \subseteq P^{*}\left(S_{1}\right) \cap P^{*}\left(S_{2}\right) \leqq S_{1}^{\sim} \cap S_{2}^{\sim} \\
= & \left(S_{1} \cap S_{2}\right)^{\sim},
\end{aligned}
$$

it follows that

$$
\left(S_{1}+M\right) \cap\left(S_{2}+M\right) \cong E \cap\left(S_{1} \cap S_{2}+M\right)^{\sim}=S_{1} \cap S_{2}+M,
$$

showing $\tau\left(S_{1}\right) \cap \tau\left(S_{2}\right) \subseteq \tau\left(S_{1} \cap S_{2}\right)$. The reverse inclusion is obvious. This completes the proof.

It follows immediately from Theorem 1 that if the unit ball $U$ is splittable and if $N$ is a closed splittable subspace then $\tau(N \cap U)$ is closed and coincides with $\tau(N) \cap \tau(U)$. Let us show that the same conclusion holds for a non-splittable subspace under suitable conditions.

Since by Corollary 3 splittability of $N$ is characterized by $P\left(N^{\llcorner}\right) \cong$ $N^{\perp} \cap M^{\perp}$, it follows by Corollary 1 that under the splittability of $U$, $N$ is splittable if and only if 


$$
\|Q f\|=\left\|f-M^{\perp} \cap N^{\perp}\right\| \quad\left(f \in N^{\perp}\right) .
$$

On the other hand, Corollary 1 implies $\|Q f\|=\left\|f-M^{\perp}\right\|$. Thus if the unit ball is splittable, splittability of $N$ is characterized by

$$
\left\|f-M^{\perp} \cap N^{\perp}\right\| \leqq\left\|f-M^{\perp}\right\| \quad\left(f \in N^{\perp}\right) .
$$

Lemma 4. Let $N_{1}$ and $N_{2}$ be closed subspace. Then for $\rho>0$ the following assertions are equivalent.

(a) $\left\|x-N_{1} \cap N_{2}\right\| \leqq \rho\left\|x-N_{1}\right\|\left(x \in N_{2}\right)$

(b) $\left\|f-N_{1}^{\perp} \cap N_{2}^{\perp}\right\| \leqq \rho\left\|f-N_{2}^{\perp}\right\|\left(f \in N_{1}^{\perp}\right)$

Proof. (a) means that

$$
N_{2} \cap\left(\overline{N_{1}+U}\right) \subseteq \overline{N_{1} \cap N_{2}+\rho U}
$$

which implies by polar formation

$$
\left(N_{1}^{\perp}+N_{2}^{\perp}\right) \cap U^{0} \cong N_{2}^{\perp}+N_{1}^{\perp} \cap \rho U^{0} .
$$

The last relation can be converted to

$$
N_{1} \cap\left(N_{2}^{\perp}+U^{0}\right) \subseteq N_{1}^{\perp} \cap N_{2}^{\perp}+\rho U^{0}
$$

which is nothing but (b). The reverse process can be pursued because (b) implies that $\left(N_{1}^{\top}+N_{2}^{\perp}\right) \cap U^{0}$ is weak* compact, hence by the Krein-Smulian theorem that $N_{1}^{\perp}+N_{2}^{\perp}$ is weak* closed.

CoROLLARY 4. Suppose that the unit ball is splittable. Then the following assertions for a closed subspace $N$ are equivalent.

(a) $N$ is splittable.

(b) $\|x-N \cap M\| \leqq\|x-N\|(x \in M)$

(c) $\left\|f-N^{\perp} \cap M^{\perp}\right\| \leqq\left\|f-M^{\perp}\right\|\left(f \in N^{\perp}\right)$.

Theorem 2. Let $M$ and $N$ be closed subspaces, and suppose that the annihilator $M^{\perp}$ is the range of a projection $P$ such that

$$
\|f\|=\|P f\|+\|f-P f\| \quad\left(f \in E^{*}\right) .
$$

If for some $1 \leqq \rho<2$

$$
\|x-N \cap M\| \leqq \rho\|x-N\| \quad(x \in M),
$$

then the images of the unit ball $U, N$ and $N \cap U$ under the canonical map $\tau$ from $E$ to $E / M$ are closed and

$$
\tau(N \cap U)=\tau(N) \cap \tau(U) .
$$

Proof. Closedness of $\tau(U)$ follows from (1) by Corollary 1 and 
Theorem 1. Let $Q=1-P$. Then in view of (1) relation (2) is converted by Lemma 4 to

$$
\left\|P f-M^{\perp} \cap N^{\perp}\right\| \leqq \gamma\|Q f\| \quad\left(f \in N^{\perp}\right)
$$

where $\gamma=\rho-1<1$. Then for any $f \in N^{\perp}$ and $g \in M^{\perp}$.

$$
\begin{aligned}
\left\|g-M^{\perp} \cap N^{\perp}\right\| & \leqq\left\|P f-M^{\perp} \cap N^{\perp}\right\|+\|g-P f\| \\
& \leqq \gamma\|Q f\|+\|g-P f\| \leqq\|g-f\|,
\end{aligned}
$$

showing

$$
\left\|g-M^{\perp} \cap N^{\perp}\right\| \leqq\left\|g-N^{\perp}\right\| \quad\left(g \in M^{\perp}\right),
$$

which is converted by Lemma 4 to

$$
\|x-M \cap N\| \leqq\|x-M\| \quad(x \in N) \text {. }
$$

This last relation means that the canonical map from the Banach space $N / M \cap N$ onto $\tau(N)$ has bounded inverse. Therefore $\tau(N)$ is closed. Further (4) implies

$$
N \cap(U+M) \subseteq \overline{U+N \cap M} .
$$

Let us prove that really

$$
N \cap(U+M) \subseteq U+N \cap M
$$

holds, which is equivalent to the required relation:

$$
\tau(N) \cap \tau(U) \leqq \tau(N \cap U) \text {. }
$$

Suppose for contradiction that there exists $x$ in $N \cap(U+M)$ with $(U-x) \cap N \cap M=\varnothing$. Since $N \cap M$ is a subspace, it follows that

$$
\operatorname{conv}((U-x) \cup\{0\}) \cap N \cap M=\{0\} .
$$

Since conv $((U-x) \cup\{0\})$ is closed, the last relation implies by polar formation that $(U-x)^{0}+N^{\perp}+M^{\perp}$ is weak* dense in $E^{*}$. Weak* closedness of $(U-x)^{0}+N^{\perp}+M^{\perp}$, if proved, leads to

$$
\operatorname{conv}\left(\left(U^{\sim}-x\right) \cup\{0\}\right) \cap N^{\sim} \cap M^{\sim}=\{0\}
$$

hence to a contradiction:

$$
x \notin E \cap\left(U^{\sim}+N^{\sim} \cap M^{\sim}\right)=\overline{U+N \cap M} .
$$

Now let us prove the above weak* closedness. To this end, in view of the Krein-Smulian theorem, it suffices to prove that for any $n>0$

$$
\left\{(U-x)^{0}+N^{\perp}+M^{\perp}\right\} \cap n U^{0} \subseteq \delta U^{0} \cap(U-x)^{0}+\delta U^{0} \cap N^{\perp}+M^{\perp}
$$


where $\delta$ is a constant depending on $n$. Remark that $(U-x)^{0}$ consists of all $f$ with $\|f\| \leqq \operatorname{Re} f(x)+1$. Since $x \in U+M$ implies $\left\|P^{*} x\right\| \leqq$ 1 , it follows from (1) that

$$
\begin{aligned}
& 1+\operatorname{Re} f(x)-\|f\| \\
\leqq & 1+\operatorname{Re} Q f(x)-\|Q f\| \\
& -\left\{\|P f\|\left\|P^{*} x\right\|-\left|P f\left(P^{*} x\right)\right|\right\} \\
\leqq & 1+\operatorname{Re} Q f(x)-\|Q f\| .
\end{aligned}
$$

This indicates that $Q$ makes $(U-x)^{0}$ invariant. Now take $f \in(U-$ $x)^{0}, g \in N^{\perp}$ and $h \in M^{\perp}$ with $\|f+g+h\| \leqq n$. Then by (1) $\| Q f+$ $Q g \| \leqq n$. Since $x \in N \cap(U+M)$ and $g \in N^{\perp}$,

$$
\begin{aligned}
\operatorname{Re} Q f(x) & \leqq n\|x\|-\operatorname{Re} Q g(x)=n\|x\|+\operatorname{Re} P g(x) \\
& \leqq n\|x\|+\left\|P g-N^{\perp} \cap M^{\perp}\right\|\|x-M\| \\
& \leqq n\|x\|+\left\|P g-N^{\perp} \cap M^{\perp}\right\| .
\end{aligned}
$$

Since $Q f$ belongs to $(U-x)^{0}$ as $f$, it follows that

$$
\begin{aligned}
\|Q g\| & \leqq n+\|Q f\| \leqq n+\operatorname{Re} Q f(x)+1 \\
& \leqq n(\|x\|+2)+\left\|P g-N^{\perp} \cap M^{\perp}\right\| .
\end{aligned}
$$

Then (3) applied to $g$ yields

$$
\left\|P g-N^{\perp} \cap M^{\perp}\right\| \leqq \frac{\gamma(n\|x\|+2)}{1-\gamma} \equiv \delta_{1}
$$

and consequently

$$
\left\|Q f+g-N^{\perp} \cap M^{\perp}\right\| \leqq n+\delta_{1} \equiv \delta_{2} .
$$

Since $x \in N, g \in N^{\perp}$ and $Q f \in(U-x)^{0}$,

$$
\|Q f\| \leqq \operatorname{Re} Q f(x)+1 \leqq \delta_{2}\|x\|+1 \equiv \delta_{3}
$$

and

$$
\left\|g-N^{\perp} \cap M^{\perp}\right\| \leqq \delta_{2}+\delta_{3} \equiv \delta
$$

This implies that

$$
\begin{gathered}
f+g+h=Q f+g+(P f+h) \\
\subseteq \delta U^{0} \cap(U-x)^{0}+\delta U^{0} \cap N^{\perp}+M^{\perp} .
\end{gathered}
$$

This completes the proof.

Consider the sup-norm Banach space $C(X)$ of continuous functions on a compact Hausdorff space $X$. By the Riesz theorem its dual is realized by the space of regular Borel measures on $X$ with totalvariation norm. Given a closed subset $Y$ of $X$, let $M$ be the subspace 
of functions in $C(X)$ vanishing on $Y$. Then $M^{\perp}$ is the set of measures with support in $Y$ and becomes the range of a projection $P: P m=$ $\chi m$ for each measure $m$ where $\chi$ is the characteristic function of $Y$. Obviously (1) is satisfied. Now let $N$ be a closed subspace of $C(X)$. As shown in [5] (3) is equivalent to the property that for any $x \in N$ with $|x(t)|<1(t \in Y)$ and any closed subset $Z \subset X$ with $Y \cap Z=\varnothing$ there is $y \in N$ such that $x(t)=y(t)(t \in Y),|y(s)|<\gamma(s \in Z)$ and $\|y\|<$ $\max (1, \gamma)$. Remark that $\|x-M\|$ coincides with the norm of the restriction $x \mid Y$ of $x$ to $Y$ and that $x(t)=y(t)(t \in Y)$ is equivalent to $x-y \epsilon$ $M$. Thus Theorem 2 shows that if (3) with $\gamma<1$, or equivalently (2) with $\rho<2$, is satisfied then for any $x \in N$ there is $y \in N$ such that $x|Y=y| Y$ and $\|y\|=\|x \mid Y\|$. The case $\gamma=0$ is the generalized Carleson-Rudin theorem (cf. [6] Chap. II). As Gamelin [5] shows, Theorem 2 can further yield the following: suppose that (3) with $\gamma<1$, or equivalently (2) with $\rho<2$, is satisfied and that $p \in C(X)$ satisfies $p(t)=1(t \in Y)$ and $p(s)>\gamma(s \varepsilon X)$. Then if $x \in N$ satisfies $|x(t)| \leqq$ $p(t)(t \in Y)$ there is $y \in N$ such that $x(t)=y(t)(t \in Y)$ and $|y(s)| \leqq$ $p(s)(s \in X)$. The case $\gamma=0$ is the Bishop theorem (cf. [6] Chap. II). Generalization of the Gamelin theorem to other direction is treated by Alfsen and Hirsberg [1].

3. Ordered Banach spaces. Let $E$ be an ordered Banach space with positive cone $E_{+}$. A closed subspace $M$ is called an ideal if ( $M-$ $\left.E_{+}\right) \cap\left(M+E_{+}\right) \subseteq M$. An ideal $M$ is hypostrict if its annihilator $M^{\perp}$ is the range of a projection $P$ such that $f \geqq P f \geqq 0$ for every $f \geqq 0$ The requirement means that both $P$ and $Q=1-P$ are positive. Perdrizet [9] shows that a closed subspace $M$ is a hypostrict ideal if and only if the following two conditions are satisfied: (1) Given $x_{1}$, $x_{2} \in M$ and $y \in E$ with $x_{1}, x_{2} \leqq y$, for any $\varepsilon>0$ there is $z \in M$ such that $x_{1}, x_{2} \leqq z \leqq y+\varepsilon$, and (2) given $x \in M$ and $y_{1}, y_{2} \in E_{+}$with $x \leqq$ $y_{1}+y_{2}$ there are $x_{1}, x_{2} \in M$ such that $x=x_{1}+x_{2}$ and $x_{i} \leqq y_{i}+\varepsilon i=$ 1,2. Under the Riesz interpolation property an ideal $M$ is hypostrict if and only if it is positively generated in the sense: $M=M \cap E_{+}$ $M \cap E_{+}$.

When $M$ is an ideal, the Banach space $E / M$ is preordered by the cone $\tau\left(E_{+}\right)$where $\tau$ is the canonical map from $E$ to $E / M$. The following theorem was first proved by Davies [4] under the Riesz interpolation property and then by Perdrizet [9] in general case. Let us give a proof based on Theorem 1 .

THEOREM 3. Let $E$ be an ordered Banach space with positive cone $E_{+}$. If $M$ is a hypostrict ideal then $E / M$ is an ordered Banach space with $\tau\left(E_{+}\right)$as its positive cone. If $E$ is regular in addition, so is $E / M$. 
Proof. Since hypostrictness means that $E_{+}^{*}=-E_{+}^{0}$ is invariant under both $P$ and $Q, E_{+}$is splittable by Corollary 3. Then $\tau\left(E_{+}\right)$is closed by Theorem 1. $M^{\perp}$ is isometric to the dual of $E / M$, and the dual positive cone is identified with $M^{\perp} \cap E_{+}^{*}$. Suppose that $E$ is regular. Then $E^{*}$ is regular. Since $P$ is positive and is of norm one in this case, $M^{\perp}$ is a regular ordered Banach space with $M^{\perp} \cap E_{+}^{*}$ as its positive cone. Therefore $E / M$ is regular as stated in $\S 1$. This completes the proof because every ordered Banach space admits an equivalent regular norm.

Corollary 5. Suppose that the positive cone $E_{+}$has nonempty interior and that $M$ is a hypostrict ideal. If a closed subspace $N$ is splittable and if it contains an interior point of $E_{+}$then $\tau\left(N \cap E_{+}\right)$ is closed and $\tau\left(N \cap E_{+}\right)=\tau(N) \cap \tau\left(E_{+}\right)$.

Proof. Since $E_{+}$is splittable, in view of Corollary 2 and Theorem 1 it suffices to prove that $\left(N \cap E_{+}\right)^{0}=N^{\perp}+E_{+}^{0}$. Remark that $f$ belongs to $\left(N \cap E_{+}\right)^{0}$ if and only if the restriction of $-f$ to $N$ is positive. However it is known (cf. [10] Chap. V §5) that when $N$ contains an interior point of $E_{+}$every continuous positive linear functional on $N$ admits a continuous positive linear extension to $E$, in other words, $-\left(N \cap E_{+}\right)^{0}=-\left(N^{\perp}+E_{+}^{0}\right)$.

Since $E / M$ is ordered by the cone $\tau\left(E_{+}\right)$, for any $y$, $z$ with $\tau(z) \leqq$ $\tau(y)$ there is $y^{\prime}$ such that $z \leqq y^{\prime}$ and $\tau(y)=\tau\left(y^{\prime}\right)$. The next task is to treat the case $\tau(z) \leqq \tau(y) \leqq \tau(x)$ and $z \leqq x$ and to find a condition of existence $y^{\prime \prime}$ such that $z \leqq y^{\prime \prime} \leqq x$ and $\tau(y)=\tau\left(y^{\prime \prime}\right)$.

Lemma 5. Let $S_{1}$ and $S_{2}$ be closed convex subsets containing 0 . If for any $0<\lambda<1, f \in S_{1}^{\prime}$ and $g \in S_{2}^{0}$ there are $f^{\prime} \in S_{1}^{0}$ and $g^{\prime} \in S_{2}^{0}$ such that

$$
\lambda f+(1-\lambda) g=\lambda f^{\prime}+(1-\lambda) g^{\prime}
$$

and

$$
\lambda\left\|f^{\prime}\right\|,(1-\lambda)\left\|g^{\prime}\right\| \leqq\|\lambda f+(1-\lambda) g\|
$$

then $\left(S_{1} \cap S_{2}\right) \sim$ coincides with $S_{1}^{\sim} \cap S_{2}^{\sim}$ where $(\cdot)^{\sim}$ denotes the weak** closure.

Proof. In view of the Krein-Smulian theorem it suffices to prove that for any $\gamma>0$ the weak* closure of conv $\left(S_{1}^{0} \cup S_{2}^{0}\right) \cap \gamma U^{0}$ is contained in the norm closure of conv $\left(S_{1}^{0} \cup S_{2}^{0}\right)$. Suppose that $0<\lambda_{\alpha}<$ $1, f_{\alpha} \in S_{1}^{0}, g_{\alpha} \in S_{2}^{0}$ and $\left\|\lambda_{\alpha} f_{\alpha}+\left(1-\lambda_{\alpha}\right) g_{\alpha}\right\| \leqq \gamma$ and that the net $\left\{\lambda_{\alpha} f_{\alpha}+\right.$ 
$\left.\left(1-\lambda_{\alpha}\right) g_{\alpha}\right\}$ weak ${ }^{*}$ converges to $h$ and the net $\left\{\lambda_{\alpha}\right\}$ converges to $\lambda$. By hypothesis $\left\{\lambda_{\alpha} f_{\alpha}\right\}$ and $\left\{\left(1-\lambda_{\alpha}\right) g_{\alpha}\right\}$ can be assumed to be bounded, hence to weak* converge to $f^{\prime}$ and $g^{\prime}$ respectively. If $0<\lambda<1,\left\{f_{\alpha}\right\}$ and $\left\{g_{\alpha}\right\}$ can be assumed to weak ${ }^{*}$ converge to $f^{\prime \prime} \in S_{1}^{0}$ and $g^{\prime \prime} \in S_{2}^{0}$ respectively. Then $h=\lambda f^{\prime \prime}+(1-\lambda) g^{\prime \prime}$ belongs to conv $\left(S_{1}^{0} \cup S_{21}^{0}\right)$. In case $\lambda=0, h=f^{\prime}+g^{\prime \prime}$ and $n f^{\prime}$ belongs to $S_{1}^{0}$ for any $n>0$. Therefore $h$, as the norm limit of $1 / n\left(n f^{\prime}\right)+(1-1 / n) g^{\prime \prime}$, belongs to the norm closure of conv $\left(S_{1}^{0} \cup S_{2}^{0}\right)$. The case $\lambda=1$ is treated similarly.

COROLLARY 6. $\quad\left(\bigcap_{i=1}^{n}\left(x_{i}-E_{+}\right)\right)^{\sim}=\bigcap_{i=1}^{n}\left(x_{i}-E_{+}\right)^{\sim}$ whenever $x_{i} \geqq$ $0 i=1,2, \cdots, n$.

Proof. $E$, hence $E^{*}$, can be assumed to be regular. $\left(x_{i}-E_{+}\right)^{0}$ consists of all $0 \leqq f$ with $f\left(x_{i}\right) \leqq 1$. Suppose that $\lambda_{i} \geqq 0, \sum_{i=1}^{n} \lambda_{i}=$ 1 and $f_{i} \in\left(x_{i}-E_{+}\right)^{0}$. Since the norm is monotone on the dual positive cone by regularity, it follows that $\lambda_{j}\left\|f_{j}\right\| \leqq\left\|\sum_{i=1}^{n} \lambda_{i} f_{i}\right\| j=$ $1,2, \cdots, n$. Now inductive application of Lemma 5 yields the assertion.

The following theorem was proved by Perdrizet [9]. Let us give a proof based on Theorem 1 .

THEOREM 4. Let $E$ be an ordered Banach space with positive cone $E_{+}$. Suppose that $M$ is a hypostrict ideal and $E / M$ is ordered by the cone $\tau\left(E_{+}\right)$where $\tau$ is the canonical map from $E$ to $E / M$. If $z_{i} \leqq 0 \leqq x_{i}$ and $\tau\left(z_{i}\right) \leqq \tau(y) \leqq \tau\left(x_{i}\right) i=1,2, \cdots, n$, then for any $\varepsilon>0$ there is $y^{\prime}$ such that $z_{i} \leqq y^{\prime} \leqq x_{i}+\varepsilon i=1,2, \cdots, n$ and $\tau(y)=\tau\left(y^{\prime}\right)$. Further $\varepsilon$ can be made 0 if every $x_{i}$ is an interior point of $E_{+}$or if $E$ has the Riesz interpolation property.

Proof. $E$ is assumed to be regular, hence $Q$ is of norm one. $z_{i}+E_{+}$is a closed convex set containing 0 . It is splittable because both $P^{*}$ and $Q^{*}$ are positive and $z_{i}$ is negative. Similary $x_{i}-E_{+}$is splittable. Let $S_{1}=\bigcap_{i=1}^{n}\left(z_{i}+E_{+}\right)$and $S_{2}=\bigcap_{i=1}^{n}\left(x_{i}-E_{+}\right)$. Then by Corollaries 2,6, and Theorem 1 both $S_{1}$ and $S_{2}$ are splittable and

$$
\begin{aligned}
& \bigcap_{i=1}^{n} \tau\left(z_{i}+E_{+}\right) \cap \bigcap_{i=1}^{n} \tau\left(x_{i}-E_{+}\right) \\
= & \tau\left(S_{1}\right) \cap \tau\left(S_{2}\right) \leqq \tau\left(S_{1} \cap\left(S_{2}+\varepsilon U\right)\right)
\end{aligned}
$$

which is just the first assertion.

If every $x_{i}$ is an interior point of $E_{+}, S_{2}$ contains 0 in its interior and by Corollary 2 and Theorem 1

$$
\tau\left(S_{1}\right) \cap \tau\left(S_{2}\right)=\tau\left(S_{1} \cap S_{2}\right) .
$$


Suppose finally that $E$ has the Riesz interpolation property. Since $E^{* *}$ becomes a lattice as stated in $\S 1, S_{1}^{\sim}$ consists of all $w \in E^{* *}$ with $\bigvee_{i=1}^{n} z_{i} \leqq w$, where $\bigvee_{i=1}^{n} z_{i}$ denotes the supremum of $z_{1}, \cdots, z_{n}$ in $E^{* *}$. Then $S_{1}^{0}$ consists of all $0 \geqq f$ with $f\left(\bigvee_{i=1}^{n} z_{i}\right) \leqq 1$. Similarly $S_{2}^{0}$ consists of all $0 \leqq g$ with $g\left(\bigwedge_{i=1}^{n} x_{i}\right) \leqq 1$ where $\bigwedge_{i=1}^{n} x_{i}$ denotes the infimum of $x_{1}, \cdots x_{n}$, in $E^{* *}$. Take $0<\lambda<1, f \in S_{1}^{0}$ and $g \in S_{2}^{0}$ and let $h=$ $\lambda f+(1-\lambda) g$. Since $E^{*}$ is a Banach lattice as stated in $\S 1$, and since both $-f$ and $g$ are positive, it follows that $0 \geqq h \wedge 0 \geqq \lambda f$ and $0 \leqq h \vee 0 \leqq(1-\lambda) g$. Let $f^{\prime}=(1 / \lambda)(h \wedge 0)$ and $g^{\prime}=(1) /(1-\lambda) h \vee$ 0 . Then it follows from the above characterization of $S_{i}^{0}$ that $f^{\prime} \in$ $S_{1}^{0}, g^{\prime} \in S_{2}^{0}$ and $h=\lambda f^{\prime}+(1-\lambda) g^{\prime}$. Now since $\|h \wedge 0\|,\|h \vee 0\| \leqq\|h\|$, Lemma 5 yields $\left(S_{1} \cap S_{2}\right)^{\sim}=S_{1}^{\sim} \cap S_{2}^{\sim}$ and the assertion follows from Theorem 1.

4. Linear lifting. Let $E$ be a Banach space with unit ball $U$ and $M$ a closed subspace. The canonical map from $E$ to $E / M$ is denoted by $\tau$. A continuous linear map $\varphi$ from $E / M$ to $E$ is called a linear lifting if $\tau \circ \phi=1$. If $\varphi$ is a linear lifting, $\varphi \circ \tau$ is a projection with $M$ as its kernel. Conversely, a linear lifting exists if $M$ is the kernel of a continuous projection.

In this section it is assumed:

There is a projection $P$ from $E^{*}$ to $M^{\perp}$ such that

$$
\|f\|=\|P f\|+\|f-P f\| \quad\left(f \in E^{*}\right)
$$

and $Q$ stands for $1-P$.

Let $F$ be a finite dimensional Banach space with unit ball $V$. Consider the dual system $\left\langle F^{*} \otimes E, F \otimes E^{*}\right\rangle$ of tensor products. When $F^{*} \otimes E$ is provided with the Minkowski functional of $\left(V \otimes U^{0}\right)^{0}$ as norm, it is called the inductive tensor product of $F^{*}$ and $E$ and is denoted by $F^{*} \oslash E$. When $F \otimes E^{*}$ is provided with the Minkowski functional of conv $\left(V \otimes U^{0}\right)$ as norm, it is called the projective tensor product of $F$ and $E^{*}$ and is denoted by $F \hat{\otimes} E^{*}$. Let $B=B(F, E)$ denote the Banach space of all continuous linear maps from $F$ to $E$, provided with operator-norm. Since $F$ is finite dimensional, $B$ is isometric to the inductive tensor product $F^{*} \circlearrowright \check{\otimes}$ under the canonical correspondence. The following lemma, whose proof is found in [10] Chap. IV $\S 9$, is basic in the subsequent development.

Lemma 6. The dual of $B(F, E)$ is isometric to the projective tensor product $F \hat{\otimes} E^{*}$ while the second dual is isometric to the inductive tensor product $F^{*} \otimes E^{* *}$, hence to $B\left(F, E^{* *}\right)$.

In view of Lemma $6 B^{* *}$ is always identified with $B\left(F, E^{* *}\right)$. 
In this case the imbedding of $B$ to $B^{* *}$ is just the natural imbedding of $B(F, E)$ to $B\left(F, E^{* *}\right)$. In accordance with the terminology in $\S 1$ the weak** closure of a subset $G$ of $B$ is formed in $B\left(F, E^{* *}\right)$ and is denoted by $G^{\sim}$. When $K$ and $S$ are a subset of $F$ and a closed convex subset of $E$ containing 0 respectively, $G(K, S)$ and $\mathscr{G}(K, S \sim)$ denote the set of all $\varphi \in B$ with $\varphi(K) \subseteq S$ and the set of all $\psi \in B^{* *}$ with $\psi(K) \subseteq S^{\sim}$. Obviously $G(K, S)$ is a closed convex subset of $B$ containing 0 and its weak ${ }^{* *}$ closure is contained in $\mathscr{G}\left(K, S^{\sim}\right)$.

Corollary 6. (a) $G(V, U)^{\sim}=\mathscr{G}\left(V, U^{\sim}\right)$. (b) $\{G(H, 0) \cap G(F$, $N)\}^{\sim}=\mathscr{G}(H, 0) \cap \mathscr{G}\left(F, N^{\sim}\right)$ if $H$ and $N$ are closed subspaces of $F$ and $E$ respectively. (c) $G(K, S)^{\sim}=\mathscr{G}\left(K, S^{\sim}\right)$ if $K$ is a cone generated by a linearly independent basis $\left\{x_{1}, \cdots, x_{n}\right\}$ of $F$ and $S$ is a cone.

Proof. (a) is an immediate consequence of Lemma 6. (b) $G(F, N)^{\sim}=$ $\mathscr{G}\left(F, N^{\sim}\right)$ follows from Lemma 6 applied to $N$ instead of $E$. Since $F$ is finite dimensional, $H$ is the kernel of a projection $\sigma$. Then

$$
\begin{aligned}
& \mathscr{G}(H, 0) \cap \mathscr{G}\left(F, N^{\sim}\right)=\mathscr{G}\left(F, N^{\sim}\right) \circ \sigma=G(F, N)^{\sim} \circ \sigma \\
\subseteq & \{G(F, N) \circ \sigma\}^{\sim}=\{G(H, 0) \cap G(F, N)\}^{\sim},
\end{aligned}
$$

while the reverse inclusion is obvious. (c) Take any $\varphi$ in $\mathscr{G}\left(K, S^{\sim}\right)$ and let $y_{i}=\varphi\left(x_{i}\right) i=1,2, \cdots, n$. Since each $y_{i}$ belongs to $S^{\sim}$, there are nets $\left\{y_{i, \alpha}\right\}$ in $S$, weak ${ }^{* *}$ converging to $y_{i} i=1,2, \cdots, n$. Consider a net $\left\{\varphi_{\alpha}\right\}$ in $B$ defined by $\varphi_{\alpha}\left(x_{i}\right)=y_{i, \alpha} i=1,2, \cdots, n$. By hypothesis it is contained in $G(K, S)$ and weak** converges to $\varphi$. Thus $\mathscr{G}\left(K, S^{\sim}\right)$ is contained in $G(K, S)^{\sim}$ with the reverse inclusion is obvious.

Since $B^{*}$ is identified with the projective tensor product $F \hat{\otimes} E^{*}$ by Lemma 6 , the operators $1 \otimes P$ and $1 \otimes Q$ are considered to define projections in $B^{*}$. The adjoints of $1 \otimes P$ and $1 \otimes Q$ are realized in $B\left(F, E^{* *}\right)$ according to the following formula:

$$
(1 \otimes P)^{*} \varphi=P^{*} \circ \varphi \text { and }(1 \otimes Q)^{*} \varphi=Q^{*} \circ \varphi\left(\varphi \in B\left(F, E^{* *}\right)\right) .
$$

LEMmA 7. The annihilator $G(F, M)^{\perp}$ is the range of $1 \otimes P$.

Proof. Since $Q^{*}$ is a projection onto $M^{\sim}$, by $(5)(1 \otimes Q)^{*}$ projects $B^{* *}$ onto $\mathscr{G}\left(F, M^{\sim}\right)$, which coincides with $G(F, M)^{\sim}$ by Corollary 6 . Then $1 \otimes P$ is obviously a projection from $B^{*}$ to $G(F, M)^{\perp}$.

On the basis of Lemma 7 , a sentence " $G(K, S)$ is splittable" will always mean that $G(K, S)$ is $1 \otimes P$-splittable.

CoRollary 7. If $S$ is splittable and $G(K, S)^{\sim}=\mathscr{G}\left(K, S^{\sim}\right)$ then 
$G(K, S)$ is splittable.

\section{Proof. This follows from (5) by Lemma 1.}

The following lemma can be considered a development of a basic device in Michael and Pełczynski [8], treating linear lifting in a special case. The crucial requirement for $P$ plays a decisive role in the proof.

Lemma 8. Suppose that $S$ is splittable and $G(K, S)^{\sim}=\mathscr{G}\left(K, S^{\sim}\right)$ for a subset $K$ of $F$. If $\psi$ belongs to

$$
G(\pi(K), S) \cap G(\pi(V), U) \cap G(K, S+M) \cap G(V, U+M)
$$

where $\pi$ is a projection of $F$ to a subspace $H$, then for any $\varepsilon>0$ there is $\varphi$ in $G(K, S) \cap G(V, U)$ such that

$$
\tau \circ \varphi=\tau \circ \psi \text { and }\|(\varphi-\psi) \mid H\|<\varepsilon .
$$

Proof. Remark first of all that the requirement for $P$ means by Corollary 1 that the unit ball $U$ is splittable.

$$
\begin{aligned}
& \text { Let } \psi_{1}=\psi-Q^{*} \circ \psi \circ(1-\pi) \text {. Since } \\
& Q^{* \circ} \psi_{1}(K) \subseteq Q^{*} \circ \psi \circ \pi(K) \subseteq Q^{*}(S) \subseteq S^{\sim}
\end{aligned}
$$

and

$$
P^{*} \circ \psi_{1}(K) \subseteq P^{*} \circ \psi(K) \subseteq P^{*}(S+M) \subseteq S^{\sim}
$$

by splittability of $S, \psi_{1}$ belongs to $\mathscr{G}\left(K, S^{\sim}\right)$ by Lemma 1 , hence to $G(K, S)^{\sim}$ by hypothesis. Since $U$ is splittable and $G(V, U)^{\sim}=\mathscr{G}(V$, $\left.U^{\sim}\right)$ by Corollary 6 , the same argument shows that $\psi_{1}$ belongs also to $G(V, U)^{\sim}$. Moreover it belongs to $\{G(K, S) \cap G(V, U)\}^{\sim}$ because $G(V, U)$ is the unit ball of $B$. On the other hand, $Q^{*} \circ \psi \circ(1-\pi)$ belongs to $\mathscr{G}(H, 0) \cap \mathscr{G}\left(F, M^{\sim}\right)$, hence to $\{G(H, 0) \cap G(F, M)\}^{\sim}$ by Corollary 6. Thus $\psi$ belongs to

$$
\{G(K, S) \cap G(V, U)+G(H, 0) \cap G(F, M)\}^{\sim} \text {. }
$$

It follows that $\psi$ must be contained in the norm closure of

$$
G(K, S) \cap G(V, U)+G(H, 0) \cap G(F, M) \text {. }
$$

Therefore there is $\psi_{2} \in B$ such that $\psi-\psi_{2} \in G(H, 0) \cap G(F, M)$ and

$$
\left\|\psi_{2}-G(K, S) \cap G(V, U)\right\|<\varepsilon .
$$

Since $G(K, S) \cap G(V, U)$ is splittable by hypothesis and Corollary 7, Lemma 3 guarantees that there is $\varphi \in G(K, S) \cap G(V, U)$ such that $\varphi-\psi_{2} \in G(F, M)$ and $\left\|\varphi-\psi_{2}\right\|<\varepsilon$. Now $\psi_{2}-\psi \in G(H, 0) \cap G(F, M)$ 
implies that $\tau \circ \varphi=\tau \circ \psi$ and

$$
\left\|(\varphi-\psi)\left|H\|=\|\left(\varphi-\psi_{2}\right)\right| H\right\| \leqq\left\|\varphi-\psi_{2}\right\|<\varepsilon .
$$

Let $S$ be a closed splittable subset of $E$ and $L$ a subset of $\tau(S)$. Suppose that there is a sequence of projections $\left\{\pi_{n}\right\}$ in $E / M$ such that (1) the range $F_{n}$ of $\pi_{n}$ is of finite dimension, (2) $\left\|\pi_{n}\right\| \leqq 1$, (3) $\pi_{n} \cdot \pi_{m}=$ $\pi_{n}$ for $n \leqq m$, (4) $\pi_{n}(L) \leqq L$ and (5) $\pi_{n}$ converges strongly to the identity as $n \rightarrow \infty$.

Let $\mathscr{G}_{n}$ denote the set of all $\varphi \in B\left(F_{n}, E\right)$ with $\varphi_{\circ} \pi_{n}(L) \subseteq S$ while $G_{n}$ is the set of all $\psi \in B\left(F_{n}, E^{* *}\right)$ with $\psi \circ \pi_{n}(L) \subseteq S^{\sim}$. As before, the second dual of $B\left(F_{n}, E\right)$ is identified with $B\left(F_{n}, E^{* *}\right)$.

LEMMA 9. If the weak** closure of $G_{n}$ coincides with $\mathscr{G}_{n}, n=1$, $2, \cdots$, then there is a linear lifting $\varphi$ from $E / M$ to $E$ such that $\varphi(L) \subseteq$ $S$ and $\|\varphi\| \leqq 1$.

Proof. Let $\pi_{0}=0$ and $\varphi_{0}=0$. Assume that linear maps $\varphi_{j} \epsilon$ $B\left(F_{j}, E\right) j=0,1, \cdots, n$ have been found in such a way that $\tau \circ \varphi_{j}=$ 1 on $F_{j},\left\|\varphi_{j}\right\| \leqq 1, \varphi_{j} \circ \pi_{j}(L) \leqq S$ and $\left\|\left(\varphi_{j-1}-\varphi_{j}\right) \mid F_{j-1}\right\|<1 / 2^{j-1} j=$ $0,1, \cdots, n$. Since $F_{n+1}$ is finite dimensional by hypothesis, there is $\psi \in B\left(F_{n+1}, E\right)$ such that $\tau \circ \psi=1$ on $F_{n+1}$. Consider the map $\psi^{\prime}=$ $\varphi_{n} \circ \pi_{n}+\psi \circ\left(1-\pi_{n}\right)$ from $F_{n+1}$ to $E$. Then by assumption

$$
\psi^{\prime} \circ \pi_{n}\left(\pi_{n+1}(L)\right)=\varphi_{n} \circ \pi_{n}(L) \leqq S
$$

and in view of $\left\|\pi_{n}\right\| \leqq 1$

$$
\psi^{\prime} \circ \pi_{n}\left(V_{n+1}\right)=\varphi_{n}\left(V_{n}\right) \cong U
$$

where $V_{i}$ denotes the unit ball of $F_{i}$. Since $V_{n+1} \subseteq \tau(U)$ by Theorem 1 and $\pi_{n+1}(L) \subseteq \tau(S)$,

$$
\psi^{\prime}\left(V_{n+1}\right) \subseteq U+M \text { and } \psi^{\prime}\left(\pi_{n+1}(L)\right) \subseteq S+M \text {. }
$$

Since the weak** closure of $G_{n}$ coincides with $\mathscr{G}_{n}$ by hypothesis, Lemma 8, applied to $F_{n+1}, \pi_{n+1}(L)$ and $\pi_{n}$ instead of $F, K$ and $\pi$, yields that there is $\varphi_{n+1} \in G_{n+1}$ such that $\left\|\varphi_{n+1}\right\| \leqq 1, \tau \circ \varphi_{n+1}=1$ on $F_{n+1}$ and $\left\|\left(\varphi_{n+1}-\varphi_{n}\right) \mid F_{n}\right\|<1 / 2^{n}$, completing induction. Now the sequence $\left\{\varphi_{n} \circ \pi_{n}\right\}$ is uniformly bounded and

$$
\sum_{k=n}^{\infty}\left\|\left(\varphi_{k+1}-\varphi_{k}\right) \mid F_{n}\right\| \leqq \sum_{j=n}^{\infty} 1 / 2^{k}<\infty
$$

guaranteeing convergence of $\varphi_{k}(x)$ for every $x \in F_{n}$ as $k \rightarrow \infty$. Then $\left\{\varphi_{n} \circ \pi_{n}\right\}$ converges strongly to some map $\varphi$ from $E / M$ to $E$. Obviously $\varphi$ is a required linear lifting.

It is better to introduce some terminology before stating the main 
result on linear lifting. A Banach space $E$ is called a $\pi$-space if there is a sequence $\left\{F_{n}\right\}$ of finite dimensional subspaces such that $F_{1} \subseteq F_{2} \subseteq \cdots$ with ${\overline{\bigcup_{n=1}^{\infty}}}_{n}=E$ and each $F_{n}$ is the range of a projection of norm one. Here projections $\pi_{n}$ can be assumed to have the property that $\pi_{n} \pi_{m}=\pi_{n}$ for $n \leqq m$ and that $\pi_{n}$ converges strongly to the identity as $n \rightarrow \infty$. An ordered Banach space is called a $\Pi$-space if, in addition, projections can be chosen positive and if each $F_{n}$ has the positive cone generated by a linearly independent basis.

THEOREM 5. Suppose that the annihilator of a closed subspace $M$ is the range of a projection $P$ such that

$$
\|f\|=\|P f\|+\|f-P f\| \quad\left(f \in E^{*}\right) .
$$

If the quotient space $E / M$ becomes a $\pi$-space then there is a linear lifting of norm one, or equivalently, $M$ is the kernel of a projection of norm one.

Proof. Since the unit ball $U$ is splittable by Corollary 1, all requirements in Lemma 9 are fulfilled with $S=U$ and $L=\tau(U)$ by Corollary 7.

Corollary 8. Let $N$ and $M$ be closed subspaces and suppose that $M^{\perp}$ is the range of a projection $P$ such that $P\left(N^{\perp}\right) \subseteq N^{\perp}$ and

$$
\|f\|=\|P f\|+\|f-P f\| \quad\left(f \in E^{*}\right) .
$$

If the quotient space $N / N \cap M$ is a $\pi$-space, there is a linear lifting of norm one from $N / N \cap M$ to $N$.

Proof. In view of Theorem 5 it suffices to prove that the annihilator of $N \cap M$ in $N^{*}$ is the range of a projection $\mathscr{P}$ such that

$$
\|g\|=\|\mathscr{P} g\|+\|g-\mathscr{P} g\| \quad\left(g \in N^{*}\right) .
$$

When $N^{*}$ is identified with $E^{*} / N^{\perp}$, the annihilator of $N \cap M$ becomes the image of $(N \cap M)^{\perp}$ under the canonical map from $E^{*}$ to $E^{*} / N^{\perp}$. Since hypothesis implies splittability of $N$ by Corollary $3, N+M$ is closed by Theorem 1 so that $N^{\perp}+M^{\perp}$ is weak* closed and coincides with $(N \cap M)^{\perp}$. Therefore the annihilator of $N \cap M$ in $N^{*}$ becomes the image of $M^{\perp}$ in $E^{*} / N^{\perp}$. Since $N^{\perp}$ is invariant under $P$, there arises a natural projection $\mathscr{P}$ from $N^{*}$ to the annihilator $N \cap M$. Since by hypothesis

$$
\left\|f-N^{\perp}\right\| \geqq\left\|P f-N^{\perp}\right\|+\left\|f-P f-N^{\perp}\right\|,
$$

$\mathscr{P}^{\text {is }}$ easily seen to have the required property. 
When $E$ is the space of continuous functions on a compact set and $M$ consists of functions vanishing on a fixed closed subset, Corollary 8 was proved by Michael and Pełczynski [8].

THEOREM 6. Let $M$ be a closed subspace of an ordered Banach space E. Suppose that $M$ is the range of a projection $P$ such that $f \geqq P f \geqq 0 \quad(f \geqq 0)$ and

$$
\|f\|=\|P f\|+\|f-P f\| \quad\left(f \in E^{*}\right) .
$$

If $E / M$ is a $\Pi$-space under the canonical ordering, there is a positive linear lifting of norm one, or equivalently, $M$ is the kernel of a positive projection of norm one.

Proof. Since the positive cone $E_{+}$is splittable by Corollary 3, all requirements in Lemma 9 are fulfilled with $S=E_{+}$and $L=\tau\left(E_{+}\right)$by definition of a $\Pi$-space and Corollary 6 .

To be a $\pi$-space or a $\Pi$-space is not so severe restriction. Let us prove:

Separable complex (resp. real) $L_{p}(1 \leqq p<\infty)$ and complex (resp. real) $C(X)$ on compact metrizable $X$ are $\pi$-spaces (resp. $\Pi$-spaces).

In fact, it suffices for the first part to treat a $L_{p}$ space on a finite measure space $(\mathscr{B}, \mu)$. Since the Borel field is separable with respect to $\mu$, there is an increasing sequence $\left\{\mathscr{B}_{n}\right\}$ of finite Borel subfields such that $\bigcup_{n=1}^{\infty} L_{p}\left(\mathscr{B}_{n}\right)$ is dense in $L_{p}$ where $L_{p}\left(\mathscr{B}_{n}\right)$ is the subspace of $\mathscr{B}_{n}$-measurable functions. Each $L_{p}\left(\mathscr{B}_{n}\right)$ is finite dimensional and the conditional expection relative to $\mathscr{B}_{n}$ becomes a (positive) projection of norm one from $L_{p}$ to $L_{p}\left(\mathscr{B}_{n}\right)$ (cf. [3]). The assertion for $C(X)$ is proved in [7] by using peaked partition.

\section{REFERENCES}

1. E. M. Alfsen and B. Hirsberg, On dominated extensions in linear subspaces of $C_{c}(X)$, Pacific J. Math., 36 (1971), 567-584.

2. T. Ando, On fundamental properties of a Banach space with a cone, Pacific J. Math., 12 (1962), 1163-1169.

3. _ Contractive projections in $L_{p}$ spaces, Pacific J. Math., 17 (1966), 391-405.

4. E. B. Davies, The structure and ideal theory of the predual of a Banach lattice, Trans. Amer. Math. Soc., 131 (1968), 544-555.

5. T. Gamelin, Restrictions of subspaces of $C(X)$, Trans. Amer. Math. Soc., 112 (1964), 278-286.

6. — Uniform algebra, Prentice-Hall, Englewood Cliffs, N. J. 1969.

7. E. Michael and A. Pełczynski, Peaked partition subspaces of $C(X)$, Illinois J. Math., 11 (1967), 555-562. 
8. E. Michael and A. Pełczynski, A linear extension theorem, Illinois J. Math., 11 (1967), 563-579.

9. F. Perdrizet, Espaces de Banach ordonnés et idéaux, J. Math. Pures Appl., 49 (1970), 61-98.

10. H. H. Schaefer, Topological Vector Spaces, MacMillan N. Y., 1966.

Received October 13, 1971.

RESEARCh INStitute of APPLiEd Electricity

HOKKAIDO UNIVERSITY, SAPPORO, JAPAN 


\section{PACIFIC JOURNAL OF MATHEMATICS}

\section{EDITORS}

\section{H. SAMELSON}

Stanford University

Stanford, California 94305

C. R. Новву

University of Washington Seattle, Washington 98105

\section{J. DuGundJI}

Department of Mathematics University of Southern California Los Angeles, California 90007

RICHARD ARENS

University of California Los Angeles, California 90024

\section{ASSOCIATE EDITORS}
E. F. BECKENBACH
B. H. NeumanN
F. WOLF
K. YoSHIDA

\section{SUPPORTING INSTITUTIONS}

\author{
UNIVERSITY OF BRITISH COLUMBIA \\ CALIFORNIA INSTITUTE OF TECHNOLOGY \\ UNIVERSITY OF CALIFORNIA \\ MONTANA STATE UNIVERSITY \\ UNIVERSITY OF NEVADA \\ NEW MEXICO STATE UNIVERSITY \\ OREGON STATE UNIVERSITY \\ UNIVERSITY OF OREGON \\ OSAKA UNIVERSITY
}

\author{
UNIVERSITY OF SOUTHERN CALIFORNIA \\ STANFORD UNIVERSITY \\ UNIVERSITY OF TOKYO \\ UNIVERSITY OF UTAH \\ WASHINGTON STATE UNIVERSITY \\ UNIVERSITY OF WASHINGTON \\ $*{ }^{*}$
AMERICAN MATHEMATICAL SOCIETY
NAVAL WEAPONS CENTER
}

The Supporting Institutions listed above contribute to the cost of publication of this Journal, but they are not owners or publishers and have no responsibility for its content or policies.

Mathematical papers intended for publication in the Pacific Journal of Mathematics should be in typed form or offset-reproduced, (not dittoed), double spaced with large margins. Underline Greek letters in red, German in green, and script in blue. The first paragraph or two must be capable of being used separately as a synopsis of the entire paper. The editorial "we" must not be used in the synopsis, and items of the bibliography should not be cited there unless absolutely necessary, in which case they must be identified by author and Journal, rather than by item number. Manuscripts, in duplicate if possible, may be sent to any one of the four editors. Please classify according to the scheme of Math. Rev. Index to Vol, 39. All other communications to the editors should be addressed to the managing editor, Richard Arens, University of California, Los Angeles, California, 90024.

50 reprints are provided free for each article; additional copies may be obtained at cost in multiples of 50 .

The Pacific Journal of Mathematics is issued monthly as of January 1966. Regular subscription rate: $\$ 48.00$ a year (6 Vols., 12 issues). Special rate: $\$ 24.00$ a year to individual members of supporting institutions.

Subscriptions, orders for back numbers, and changes of address should be sent to Pacific Journal of Mathematics, 103 Highland Boulevard, Berkeley, California, 94708.

PUBLISHED BY PACIFIC JOURNAL OF MATHEMATICS, A NON-PROFIT CORPORATION

Printed at Kokusai Bunken Insatsusha (International Academic Printing Co., Ltd.), 270, 3-chome Totsuka-cho, Shinjuku-ku, Tokyo 160, Japan. 


\section{Pacific Journal of Mathematics}

\section{Vol. 44, No. $2 \quad$ June, 1973}

Tsuyoshi Andô, Closed range theorems for convex sets and linear liftings . . . . . . 393

Richard David Bourgin, Conically bounded sets in Banach spaces . . . . . . . . . 411

Robert Jay Buck, Hausdorff dimensions for compact sets in $R^{n} \ldots \ldots \ldots \ldots \ldots \ldots . \ldots 421$

Henry Cheng, A constructive Riemann mapping theorem ................ 435

David Fleming Dawson, Summability of subsequences and stretchings of

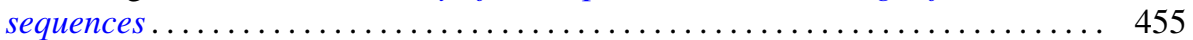

William Thomas Eaton, A two sided approximation theorem for 2-spheres ....... 461

Jay Paul Fillmore and John Herman Scheuneman, Fundamental groups of compact complete locally affine complex surfaces ....................... 487

Avner Friedman, Bounded entire solutions of elliptic equations . . . . . . . . . . . 497

Ronald Francis Gariepy, Multiplicity and the area of an $(n-1)$ continuous

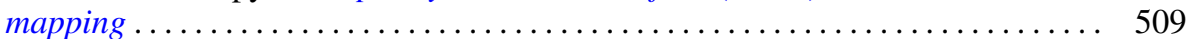

Andrew M. W. Glass, Archimedean extensions of directed interpolation groups . . . . 515

Morisuke Hasumi, Extreme points and unicity of extremum problems in $H^{1}$ on

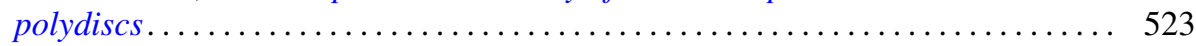

Trevor Ongley Hawkes, On the Fitting length of a soluble linear group . . . . . . 537

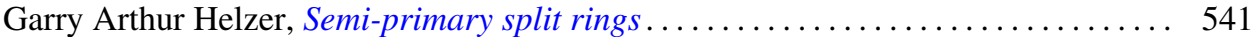

Melvin Hochster, Expanded radical ideals and semiregular ideals . . . . . . . . . 553

Keizō Kikuchi, Starlike and convex mappings in several complex variables . . . . . . 569

Charles Philip Lanski, On the relationship of a ring and the subring generated by its

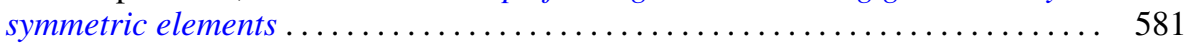

Jimmie Don Lawson, Intrinsic topologies in topological lattices and semilattices ........................................... 593

Roy Bruce Levow, Counterexamples to conjectures of Ryser and de Oliveira ...... 603

Arthur Larry Lieberman, Some representations of the automorphism group of an infinite continuous homogeneous measure algebra ..........

William George McArthur, $G_{\delta}$-diagonals and metrization theorems $\ldots .$.

James Murdoch McPherson, Wild arcs in three-space. II. An invariant of

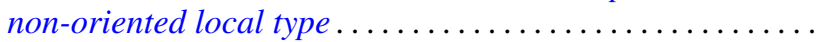

H. Millington and Maurice Sion, Inverse systems of group-valued measures ...

C. Edward Moore, Concrete semispaces and lexicographic separation of convex

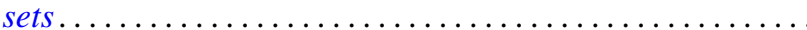

Jingyal Pak, Actions of torus $T^{n}$ on $(n+1)$-manifolds $M^{n+1}$.

Merrell Lee Patrick, Extensions of inequalities of the Laguerre and Turán type . . . . 675

Harold L. Peterson, Jr., Discontinuous characters and subgroups of finite index. . . . 683

S. P. Philipp, Abel summability of conjugate integrals . . . . . . . . . . . . . 693

R. B. Quintana and Charles R. B. Wright, On groups of exponent four satisfying an

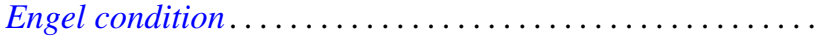

Marlon C. Rayburn, On Hausdorff compactifications. . . . . . . . . .

Martin G. Ribe, Necessary convexity conditions for the Hahn-Banach theorem in

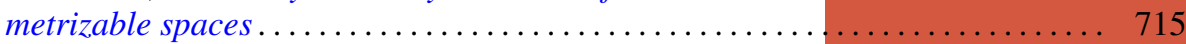

Ryōtarō Satō, On decomposition of transformations in infinite measure spaces .... 733

Peter Drummond Taylor, Subgradients of a convex function obtained from a

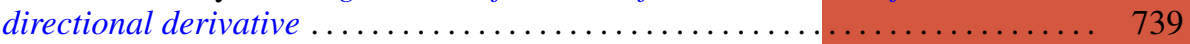

James William Thomas, A bifurcation theorem for $k$-set contractions . . . . . . . . 749 Clifford Edward Weil, A topological lemma and applications to real functions . . . . 757

Stephen Andrew Williams, A nonlinear elliptic boundary value problem . . ....... 767

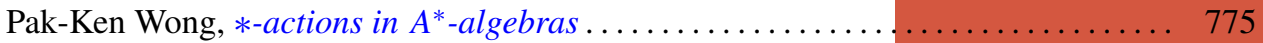

